\title{
Wer fragt riskiert Antworten
}

\author{
Erhard Taverna
}

«Pontoniere müssen lesen und schreiben können, die Sappeure noch dazu die vier Species des Rechnens mit ganzen Zahlen beherrschen. Dasselbe gilt für die Artilleristen, von denen überdies erwartet wird, dass sie sich durch Intelligenz auszeichnen.» Die Tagsatzung von 1841 erwähnt bei den Kavalleristen keine Schulkenntnisse, wohl aber Gewandtheit und Intelligenz. Die Prüfung des männlichen Nachwuchses im elementaren Schulwissen wurde zum Motor für dringend nötige Schulreformen. Die ersten pädagogischen Rekrutenprüfungen fanden 1854 im Kanton Solothurn statt. Ab 1875 wurden die Rekrutenprüfungen Bundessache und dienten de facto dazu, die Leistungen der Kantone im Schulwesen zu überwachen.

\section{Von der Kaserne ...}

Die eidgenössische Schulpolitik wurde durch die gefürchteten Prüfungen massgeblich beeinflusst. Die Noten wurden im Dienstbüchlein vermerkt, die Resultate von Gemeinden und Kantonen in Ranglisten verglichen. Für die Walliser muss es 1873 ein Schock gewesen sein, dass nahezu ein Sechstel der Rekruten weder lesen noch schreiben konnte. Die Institution kam gegen Ende des Jahrhunderts zunehmend unter Beschuss: «Wir erziehen nicht mehr Menschen, Persönlichkeiten, sondern Kontrollnummern für die Rekrutenprüfung. Unsere Schule ist nicht Schule für das Leben, sondern Schule für die Rekrutenprüfung. Die heiligste Pflicht eines bernischen Lehrers ist nicht mehr, Herz und Gemüt, Charakter und Willen seiner Zöglinge zu bilden; seine heiligste Pflicht ist, dafür zu sorgen, dass sein Heimatkanton bei der Rekrutenprüfung einen ehrenvollen Rang einnimmt.» Das jährliche Ritual erstarrte zur Schablone und blieb trotz der nachweisbaren Leistungssteigerung von 1914 bis 1929 abgeschafft. Nach der Wiedereinführung überwogen die mündlichen Prüfungen, wobei die vaterlandskundlichen Gesprächsthemen ab 1940 die Weltpolitik widerspiegeln. Nach einem Truppenbesuch 1963 in Thun schrieb Admiral Rickover, der Organisator der US-Atomunterseebootflotte, von einem unvergesslichen Erlebnis: «The recruit exam as a means to test the effectiveness of public education in a democracy strikes me as nothing short of genius.» Staats- kunde als Schulfach, Jungbürgerkurse und Prüfungen beeinflussten sich gegenseitig. 1970 stimmte der Nationalrat dem Postulat Abegg zu, das von den Prüfungen eine neue Zielsetzung verlangte, um diese «zu einem tauglichen Instrument der Meinungsforschung und der Information» zu verändern. Die einsetzende Zusammenarbeit mit Hochschulinstituten führte zwei Jahre später zu wissenschaftlichen Publikationen und 1991 zu einer ersten Längsschnittuntersuchung über traditionelle Bereiche wie Lesen, Schreiben, Rechnen, Staats- und Wirtschaftskunde. 1998/99 wurde erstmals eine ergänzende Repräsentativbefragung von 1000 Nichtrekruten und Frauen in allen Landesteilen durchgeführt.

Seit im Durchschnitt bloss noch etwa 7 von 10 männlichen 20jährigen Schweizern die RS absolvieren, erfasst das Rekrutensegment heute nur etwa 29\% der 20jährigen schweizerischen Wohnbevölkerung. Es fehlen zudem die Schweizerinnen und die jugendlichen Ausländer. Dazu kommt die gestiegene Ausfallquote in den ersten 10 RS-Tagen und ein ziviler Ersatzdienst, die die Rekrutenpopulation noch einmal verändern. Relevante Schlussfolgerungen auf der Basis von Befragungen erfordern heute zyklische Wiederholungen im Rahmen von Langzeitstudien und Stichprobenerhebungen unter allen Jugendlichen.

\section{... zum nationalen Jugendmonitoring}

Mit dem neuen, wissenschaftlichen Monitoring erhoffen sich die Forscher Antworten auf die Fragen einer wirksamen Jugend-, Gesundheits- und Sozialpolitik. Soziale Indikatorsysteme, von der OECD geplante Surveys zu sogenannten Life Skills, demoskopische Stichproben vermittels Interviews und Trendstudien sollen die nötige Datenbasis für politisches und soziales Handeln sicherstellen. Die neuen «Jugend- und Rekrutenbefragungen ch-X» zeigen bereits beachtliche Resultate. Zum Beispiel die grösste, je in einem westlichen Land durchgeführte Studie zum Thema der sexuellen Gewalt unter 20jährigen mit bemerkenswerten Erkenntnissen und Folgerungen. So steht fest, dass es sich bei Vergewaltigern um eine kleine aber hochaktive Gruppe von Mehrfachtätern handelt, dass die Opfer einem hohen Risiko für eine Ansteckung mit AIDS und 
Hepatitis C ausgesetzt sind, dass die Opfer speziell ausgebildete Strafverfolgungsbehörden benötigen, dass DNA-Datenbanken, auch von Nicht-Sexualdelinquenten, angesichts der hohen Prävalenz «normaler» Delikte unter Vergewaltigern nötig wären, und dass in therapeutischer Hinsicht ein dringender Handlungsbedarf für sexuell viktimisierte Knaben besteht. Brisante Resultate, die entschlossenes politisches Handeln erfordern! Weitere Schwerpunktthemen waren Erhebungen zu passiven und aktiven Gewalterfahrungen, zu Bildern und Wertvorstellungen und 1999 eine Querschnittserhebung zu extremistischen und männerbündischen Orientierungen.

Die alte Rekrutenprüfung hat sich zeitgemäss weiterentwickelt. Zu hoffen ist, dass unter dem Druck der Erkenntnisse auch die Politik entsprechende Fortschritte macht. Bereits 1991 hält die erwähnte Studie fest: «Im ganzen gesehen ist der Stand der Grundausbildung (lesen, schreiben, rechnen) bei den Rekruten enttäuschend. So war nur die Hälfte der jungen Männer in der Lage, aufgrund einfacher Zahlen einen Prozentsatz zu errechnen. Noch erstaunlicher: nur einem von vieren gelang es, einen diktierten Satz von vier Zeilen fehlerlos wiederzugeben, und auch nur einer auf vier scheint den Hauptgedanken eines recht einfachen Zeitungsartikels verstanden $\mathrm{zu}$ haben.» 10 Jahre später schneidet die Schweiz im internationalen Vergleich erneut schlecht ab.

Fragen drängen sich auf: sammeln wir, 127 Jahre nach der ersten eidgenössischen Rekrutenprüfung, nur Datenfriedhöfe, ohne jede Konsequenz, und würde man vielleicht besser mit dem vielzitierten «Empowerment» erst einmal hier ansetzen?

\section{Literatur}

- Lustenberger W. Pädagogische Rekrutenprüfungen. Chur/Zürich: Verlag Rüegger; 1996.

- Pädagogische Rekrutenprüfungen. Wissenschaftliche Berichte, Bände 13, 14, 15. Bericht 98/99 und Werkstattberichte 00/01. 